\title{
Uusioersily
}

\section{Piezoelectric sensor determination of arterial pulse wave velocity}

McLaughlin, JAD., McNeill, MDJ., Braun, B., \& McCormack, PD. (2003). Piezoelectric sensor determination of arterial pulse wave velocity. Physiological Measurement, 24(3), 693-702. https://doi.org/10.1088/0967$3334 / 24 / 3 / 306$

Link to publication record in Ulster University Research Portal

\section{Published in:}

Physiological Measurement

Publication Status:

Published (in print/issue): 01/01/2003

DOI:

10.1088/0967-3334/24/3/306

\section{Document Version}

Publisher's PDF, also known as Version of record

\section{General rights}

Copyright for the publications made accessible via Ulster University's Research Portal is retained by the author(s) and / or other copyright owners and it is a condition of accessing these publications that users recognise and abide by the legal requirements associated with these rights.

\section{Take down policy}

The Research Portal is Ulster University's institutional repository that provides access to Ulster's research outputs. Every effort has been made to ensure that content in the Research Portal does not infringe any person's rights, or applicable UK laws. If you discover content in the Research Portal that you believe breaches copyright or violates any law, please contact pure-support@ulster.ac.uk. 


\title{
Piezoelectric sensor determination of arterial pulse wave velocity
}

\author{
J McLaughlin $^{1}$, M McNeill ${ }^{1}$, B Braun ${ }^{1}$ and P D McCormack ${ }^{2}$ \\ ${ }^{1}$ Northern Ireland Bioengineering Centre, University of Ulster at Jordanstown, Shore Road, \\ Newtownabbey, Co Antrim, BT37 0QB, UK \\ 2 Department of Bioengineering, University of Illinois at Chicago, 851 S Morgan Street, Chicago, \\ IL 60607-7052, USA
}

Received 14 January 2003, in final form 16 April 2003

Published 4 June 2003

Online at stacks.iop.org/PM/24/693

\begin{abstract}
Arterial pulse wave velocity (APWV) is a measure of the elasticity (or stiffness) of peripheral arterial blood vessels. The pulse referred to here will be the pressure pulse as opposed to the flow pulse measured by ultrasound Doppler. The pressure pulse velocity varies over the range from about $12 \mathrm{~m} \mathrm{~s}^{-1}$ to $15 \mathrm{~m} \mathrm{~s}^{-1}$ in stiff peripheral arteries, whereas in normal arteries it has a velocity in the range of 7 to $9 \mathrm{~m} \mathrm{~s}^{-1}$.

The aim of this project was the development of a fast and easy to use system for the determination of peripheral arterial pulse wave velocity. The principle of the PWV measurement is based on simultaneous measurement of two pulse waves at two different positions, such as the radial artery at the wrist and the brachial artery just above the elbow. By determining the pulse transit time between these points and the distance measured between the two locations, pulse wave velocity may then be calculated. The pressure pulse detection is done by using two piezoelectric sensors which generate a measurable voltage at the output contacts if they are mechanically deformed. The deformation produced voltage is first amplified and filtered and then digitalized with a data acquisition card. The analysis of the data obtained from the sensors includes a filtering process, the calculation of the PWV with three different methodsfoot-to-foot, cross-correlation and peak-to-peak-and the determination of the arterial pulse rate.

Extensive measurements with human test subjects were carried out to optimize the techniques of data acquisition and analysis. For example, it was found that the best procedure was to hold the sensors in place using elastic straps alone. The data analysis was upgraded with an additional software module, which deletes, in effect, outriders or invalid measurements. With the optimized system, a series involving eight test subjects ranging in age from 22 to 32 years was completed (all normotensive). The arterial pulse wave velocities determined covered a range from $6 \mathrm{~m} \mathrm{~s}^{-1}$ to $12 \mathrm{~m} \mathrm{~s}^{-1}$, with an average standard deviation of less than $2.5 \mathrm{~m} \mathrm{~s}^{-1}$ for individual results. These are slightly higher, but close to published APWV data. The results showed that
\end{abstract}


reproducible results can be obtained with the existing PWV acquirement and analysis system.

Keywords: arterial pulse wave velocity, APWV, non-invasive, pressure pulse, piezoelectric sensor, elasticity, stiffness, peripheral

\section{Introduction}

The measurement of arterial pulse wave velocity (APWV) is one of the methods used to measure physiological changes in peripheral vascular disease. Others include pressure pulse contour, arterial elasticity, pulsatile flow, complex vascular impedance and cardiac work. There have been many investigations over the past 30 years or so to relate changes in age and progress of arterial disease, to vessel pathology and distensibility based on the propagation characteristics of the arterial pulse (Malindzak and Meredith 1970, McCormack 1981, Persson et al 2001, Ramsey 1995, Wilkinson 1998). They have only been partially successful from a clinical point of view, mainly due to the difficulty in controlling the effects of patient parameters (autonomic system, patient movement, etc).

Early on, the methods of determining APWV involved direct invasive measurement of the pulse pressure at two points along an arterial segment and measuring the time taken for the pulse to travel the length of the segment. Such investigations had to be limited to animals, usually dogs. Malindzak and Meredith (1970) carried out a comparative study of the arterial pulse wave velocity on anaesthetized dogs by recording intra-luminal pressure measured at two sites (proximal and distal) along the abdominal aorta axis.

Non-invasive methods have included flow Doppler (McCormack 1981) and tissue Doppler (Persson et al 2001) sensors using human test subjects. The comparative study referred to above was required because the pulse velocity measurement can be significantly modified, and differs from the true pulse velocity (TAPWV) due to the viscosity of the fluid, the presence of reflected components (from bifurcations, stenoses and peripheral vascular beds) and due to changes in the elastic composition of the arterial wall. All of these lead to alterations in the pulsatile wave characteristics, so that what is measured is an apparent pulse wave velocity (AAPWV). The true PWV is that velocity of the arterial pressure pulse at which pressure waves would propagate in an infinite homogeneous tube (with a length much greater than the wavelength).

To reliably predict the elastic parameters ('stiffness') of the arterial wall, which are of fundamental importance to the understanding of the mechanics of the arterial system, depends on getting an accurate measure of the true arterial pulse wave velocity. Modelling the peripheral arterial segment as a thin-walled isotropic, incompressible tube containing an incompressible, inviscid fluid, then the PWV is related to the wall elasticity by the Moens-Kortweg equation (Moens 1878),

$$
\mathrm{c}_{0}=\sqrt{ }(E h / 2 R \rho)
$$

where $h$ is the wall thickness, $R$ is the lumen radius, $E$ is the wall elasticity modulus and $\rho$ is the fluid (blood) density.

\subsection{Methods}

There are four basic methods for the determination of the arterial PWV. 
1.1.1. 'Foot-to-foot' APWV (FFAPWV). This is based on the velocity of the 'foot', or leading edge, of the pressure pulse wave. The arrival times of the foot of the pulse wave at two positions along the artery are recorded. If $\Delta t$ is the difference in arrival times and $\Delta s$ the distance between the two recording positions (proximal and distal), the FFAPWV is simply

$\mathrm{FFAPWV}=\Delta s / \Delta t$

1.1.2. 'Peak-to-peak' APWV (PPAPWV). This is completely analogous to the FFAPWV except that the points of observation are the two (proximal and distal) peaks of the pulse wave and

$$
\operatorname{PPAPWV}=\Delta s / \Delta t
$$

The PPAPWV is particularly sensitive to wave reflections along the course of the artery.

1.1.3. Apparent pulse wave velocity $(A A P W V)$. The pressure wave may be represented as a Fourier series,

$$
P(t)=P_{0}+\sum_{n=1}^{k} P_{n} \sin \left(n \omega t+\theta_{n}\right)
$$

where $P_{0}$ is the mean fluid pressure, $n$ is the harmonic number, $P_{n}$ is the amplitude of the $n$th harmonic and $\theta_{n}$ is the phase angle of the $n$th harmonic.

The spatial rate of change of the phase for one harmonic based on two simultaneous pressure measurements separated by a distance $\Delta s$ along an artery, is related to the apparent arterial pulse wave velocity (AAPWV) by the following equation,

$$
\operatorname{AAPWV}_{n}=(\Delta s) n(f)\left(360^{\circ}\right) /\left(\theta_{x_{1}}-\theta_{x_{2}}\right)
$$

where $\mathrm{AAPWV}_{n}$ is the apparent pulse wave velocity for the $n$th harmonic, $f$ is the heart rate, $\theta_{x_{1}}$ is the phase angle for the proximal harmonic $n$ and $\theta_{x_{2}}$ is the phase angle for the distal harmonic $n$.

The presence of reflected wave components within the arterial system can appreciably modify the amplitude and phase angle of the Fourier components of the pulse wave. So the PWV determined differs from the true value of the PWV, and hence the use of the term 'apparent'.

1.1.4. Cross-correlation $P W V$. If the arterial pulse at the proximal measurement position is represented by the pressure time series $P\left(x_{1}, t\right)$ and that at the distal position by $P\left(x_{2}, t\right)$ and the cross-correlation coefficient is $\Phi_{x_{1}, x_{2}}(\tau)$, then $\Phi$ will have a maximum value at some time lag, $\tau$ (see figure 1).

The correlation function can be expressed as

$$
\Phi_{x_{1}, x_{2}}(\tau)=(1 / T) \int_{-\frac{T}{2}}^{\frac{T}{2}} P\left(x_{1}, t\right) P\left(x_{2}, t\right) \mathrm{d} t .
$$

The value of $\tau$ at which maximum correlation occurs represents the transit time $(\Delta t)$ of the pressure wave from position $x_{1}$ to position $x_{2}$ along the arterial segment. From the separation distance and transit time data the correlation arterial pulse wave velocity is

$$
\mathrm{CCAPWV}=\left(x_{2}-x_{1}\right) / \Delta t .
$$


CROSS CORRELATION PROPAGATION VELOCITY
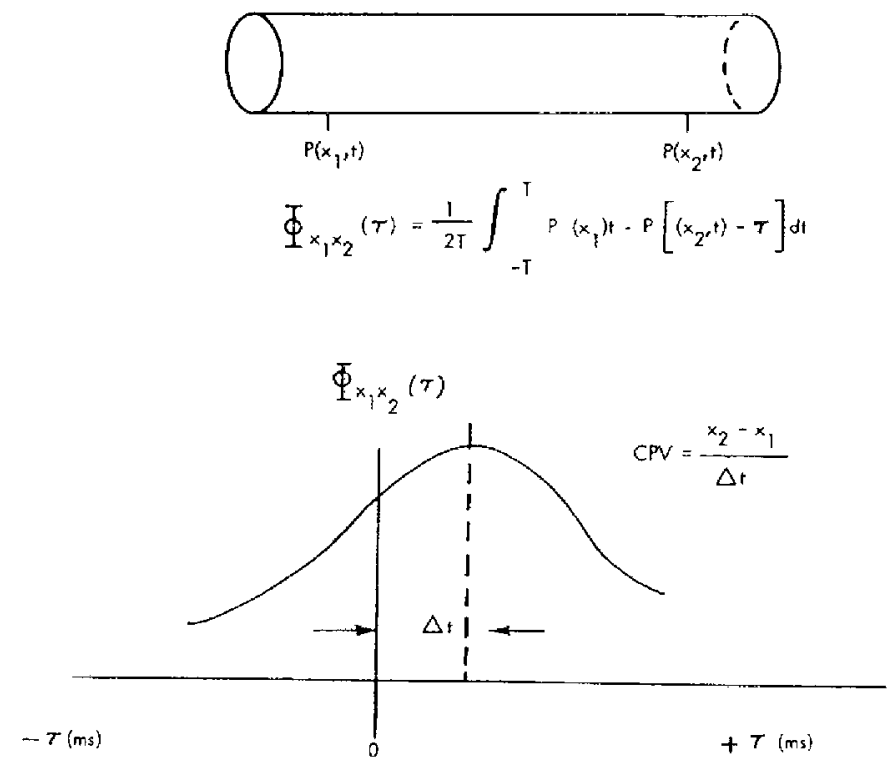

Figure 1. Cross-correlation propagation velocity. $x_{1}$ : proximal arterial position; $x_{2}$ : distal arterial position.

\section{Outline of program}

The sensor technique used in this work involves the piezoelectric effect in polyvinylidene fluoride (PVDF), which produces an output voltage in response to mechanical pressure on the material. Sensor details and the associated signal acquisition and data analysis will be presented in section 4 .

Three methods of APWV determination are used: foot-to-foot APWV; peak-to-peak APWV and cross-correlation APWV. The FFAPWV and CCAPWV methods are less sensitive to pressure wave reflections at bifurcations, etc in the arterial tree, than the PPAPWV method. Mean values and standard deviations were computed for all three methods and compared.

In this work normal, young test subjects were used, and it has the primary objectives of optimizing the measurement procedures and establishing the statistical spread and mean values of the observed PWVs for a specific peripheral arterial segment. Based on this, it is planned to use the system in clinical trials involving patients with peripheral arterial disease (due to diabetes, hypertension, etc), pre-, during and post treatment (pharmaceutical or surgical).

\section{Experimental details}

\subsection{Polyvinylidene fluoride (PVDF) thin film piezoelectric sensor system}

Piezoelectric materials convert mechanical stress or strain into proportionate electrical energy. Piezo film has relatively high output voltage_about ten times greater than ceramic materials. It is a thin, flexible, lightweight, mechanically tough plastic film. It has a wide frequency range $\left(0.001 \mathrm{~Hz}\right.$ to $\left.10^{9} \mathrm{~Hz}\right)$, low acoustical impedance, moisture resistance and can be glued with commercial adhesives. 


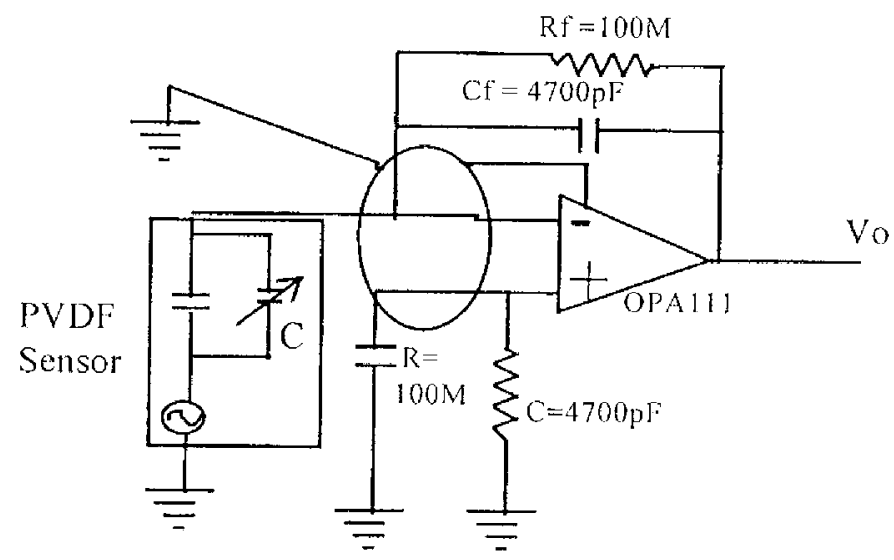

Figure 2. PVDF charge amplifier.

The low acoustic impedance when close to human tissue permits more efficient transduction of acoustic signals in tissue. The PVDF material is a thin plastic polymer that has a thin electrically conductive nickel copper alloy deposited on each side. Wire connections are made to the piezo film sensor. Two important parameters of these films are:

(i) piezoelectric strain constant $=23 \times 10^{-12} \mathrm{~m} \mathrm{~V}^{-1}$,

(ii) piezoelectric voltage constant $=216 \times 10^{-3} \mathrm{~m} \mathrm{~N}^{-1}$.

\subsection{Analogue and digital circuitry}

3.2.1. Analogue charge amplifier. Piezoelectric materials convert mechanical stress or strain into proportionate electrical energy, by producing a charge when subjected to mechanical stress. The charge is converted to a voltage by an operational amplifier connected as a current integrator, called a charge amplifier (figure 2). The signal output of the amplifier is approximately $-30 \mathrm{mV}$. It is augmented by signal amplification.

3.2.2. Analogue signal amplification. This is done by use of an inverting amplifier. Because a dc signal appears at the output of the charge amplifier, dc offset removal is essential and is implemented in the inverting summing amplifier shown in figure 3 .

The next phase of the analogue circuitry is a low pass filter to remove the $50 \mathrm{~Hz}$ noise interference (see figure 4).

3.2.3. Digital controlled data acquisition and analysis. A data acquisition board (DAQ) is required when the transducer signals need interfacing with a PC. The board contains 12 bit plus sign and a successive approximation and self-calibrating analogue-to-digital (ADC) converter. The ADC incurs a systematic error known as the quantization error. It is due to limited resolution and with the analogue input limit set at -5 to $+5 \mathrm{~V}$, the quantization error of the A/D converter used here was calculated to be $0.122 \mathrm{mV}$.

In this system measurements were made with respect to earth potential and so the mode is named reference single ended (RSE).

The data acquisition and analysis was done using LabView—a powerful instrumentation and analysis programming language for PCs. 


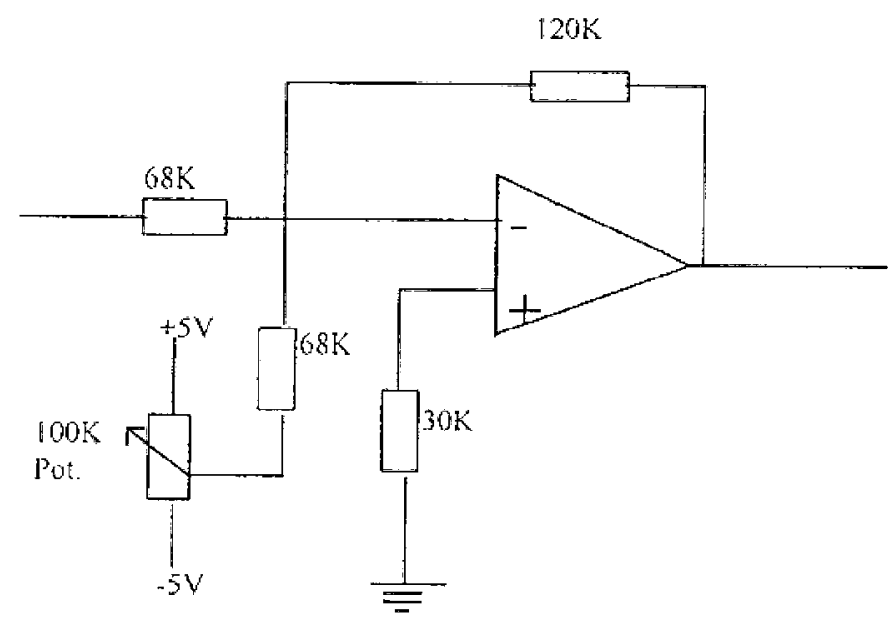

Figure 3. Inverting summing amplifier.

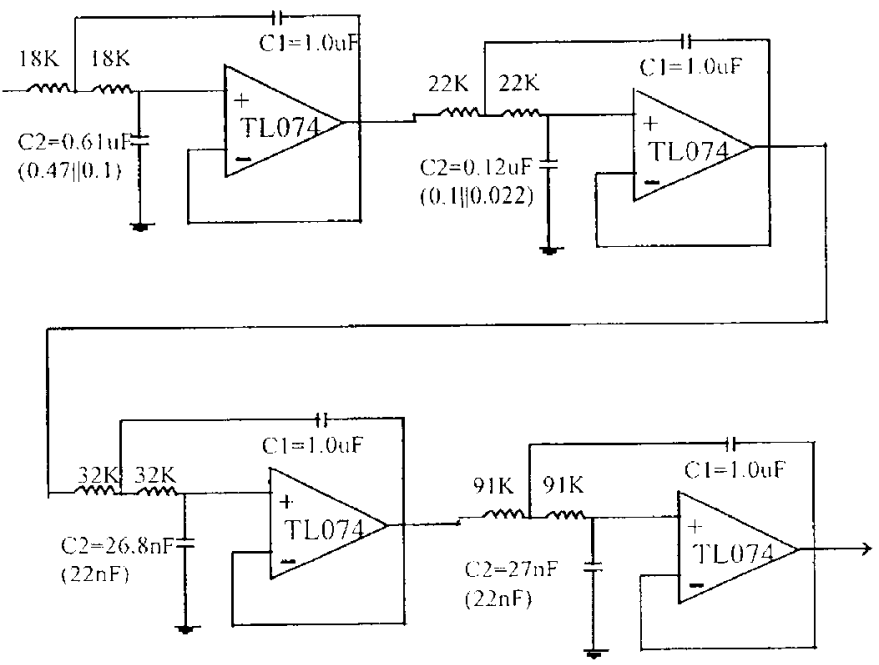

Figure 4. Chebyshev filter.

3.2.4. Digital data acquisition program. The data acquisition circuit performs all the necessary operations for the data acquisition with LabView. The functions of the circuit initialize the data acquisition and read the data from the acquisition card. These data are stored for later use in the data analysis part of the program.

LabView programs are called virtual instruments (VIs) because of their appearance and operations are analogous to measuring instruments. A VI that is called within another VI is called a sub-VI and is analogous to a subroutine in text-based languages.

The data acquisition, analysis and presentation are comprised of three main procedures:

(1) Data acquisition card to interface the hardware to the PC.

(2) Data acquisition program to acquire and store data in a spreadsheet file.

(3) Data analysis to carry out digital signal processing, calculate PWV and present results. 
$P W V$ calc VI. In this sub-VI the PWV is calculated from the locations of peaks in the signals of sensors $\mathrm{A}$ and $\mathrm{B}$ belonging to it:

$$
\operatorname{PWV}\left(\mathrm{m} \mathrm{s}^{-1}\right)=\frac{\text { location of peak in sensor B }- \text { location of peak in sensor A }}{\text { sampling frequency }} \text {. }
$$

Pulse Rate VI. The input is an array of the location of peaks and the sampling frequency, and the output is the pulse rate:

Pulse rate $\left(\right.$ beats $\left.\min ^{-1}\right)=\frac{60 \times \text { sampling frequency }}{\text { location of peak (element } 1)- \text { location of peak (element } 0)}$.

Reflect VI. This VI computes the reflection index (RI) and the late systolic (or reflection) time lag.

\subsection{PWV Calculation}

3.3.1. PWV calculation using peak detection. To calculate PWV using peaks, the location of the peaks must first be determined, so that the transit time of the wave between the peaks can be determined. It was found that the best method of peak detection is the derivative of the curve method. If the first derivative of a curve is zero, then an extreme value can exist-either a peak or a turning point. It is necessary to take the second derivative at this point-if this is also zero, then an extreme value exists. The second VI used to determine the PWV is PWVcalc, using the time separation between the located peaks.

3.3.2. PWV with pressure wave foot detection. The VI named PWV Foot determines the leading edge (foot) of the pressure wave at the upstream and downstream locations. The VI named PWVcalc is again used to compute the PWV from the time separation between the two leading edges ('foot-to-foot' APWV or FFAPWV).

3.3.3. PWV with cross-correlation. The PWV determination with cross-correlation is done with the VI named CalcPWV. The VI is in two parts: a part for the initialization function and a part for the calculation of the CCAPWV.

In all cases PWV values are assembled in an array and the mean value, standard deviation and variance are calculated.

\subsection{Sensor positioning}

Sensor placement is critical to obtaining consistent measurements. A screw mechanism was first used to apply the sensors to the skin. But readings were very variable and so this technique was replaced by that in which the sensors are fixed to the skin by elastic strips. This led to better results.

Figure 5 shows the sensor positioning technique using the elastic bandage which hides the sensors from view.

Arm position is another critical feature of measurement. Two positions, normal and dependent, were analysed in detail, using one test subject. In the normal position, the subject sits with the arm resting on a table. All test subject measurements were made in this position (see table 1). In the dependent position, the subject sits with the arm hanging straight down. The PWV values were smaller in this position and the variability was much greater, so this technique was discarded.

A correction module, 'Delete Incorrect', was used to delete PWV values resulting from aberrant signals, caused commonly by arm movement during the measurement. These unrealistic values were deleted before the PWV values were passed to the analysis VI. 


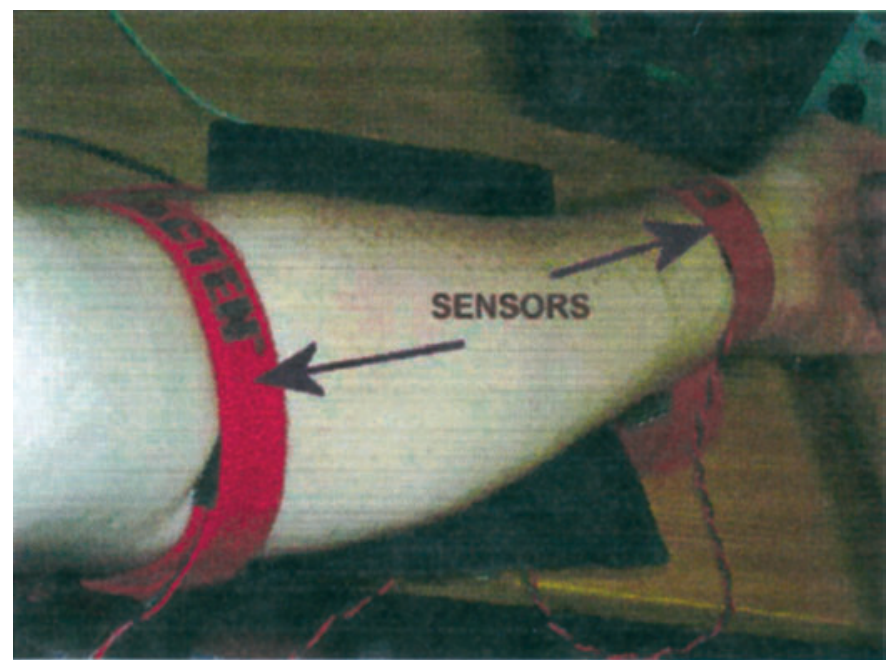

Figure 5. Sensor positioning with elastic straps.

(This figure is in colour only in the electronic version)

Table 1. PWV measured in normal arm position.

\begin{tabular}{lllll}
\hline & \multicolumn{4}{c}{ PWV $\left(\mathrm{m} \mathrm{s}^{-1}\right)$} \\
\cline { 2 - 5 } Measurement & PPAPWV & CCAPWV & FFAPWV & Mean value \\
\hline 1 & 5.7 & 7.74 & 8.87 & 7.44 \\
2 & 5.83 & 7.49 & 8.69 & 7.34 \\
3 & 6.87 & 7.20 & 6.89 & 6.99 \\
4 & 5.78 & 6.98 & 7.98 & 6.91 \\
\hline
\end{tabular}

\section{Experimental results}

A preliminary trial series will be reported here, with the objectives of comparing the various methods of computing the PWV and optimizing the measurement techniques in terms of reproducibility of the results.

The test series involved eight subjects, all normotensives, on no medication, with no heart disease and in the age range from 22 to 32 years. It took about three weeks to complete and at least 12 measurement days on each of the test subjects were achieved.

Figure 6 shows arterial pulse wave velocity results for subject 1 (typical) over 12 days of measurement. The mean APWV over the three methods of measurement (PP, FF and CC) varies between $6 \mathrm{~m} \mathrm{~s}^{-1}$ and $10 \mathrm{~m} \mathrm{~s}^{-1}$, with an overall mean of $7.25 \mathrm{~m} \mathrm{~s}^{-1}$.

Figure 7 is a histogram presentation for each of the eight subjects, of the APWV values from the three calculation methods plus the overall mean.

Figure 8 is a plot of overall mean APWV values for the eight test subjects, plus and minus the standard deviations of the mean.

The overall mean APWV for this age group is about $8.2 \mathrm{~m} \mathrm{~s}^{-1}$ with a mean standard deviation of about $1 \mathrm{~m} \mathrm{~s}^{-1}$ This is consistent with a recent publication (Persson et al 2001), which reported the results of an 'improved tissue Doppler method', of $7.4 \mathrm{~m} \mathrm{~s}^{-1}$ with a mean standard deviation of $1 \mathrm{~m} \mathrm{~s}^{-1}$, and with other recently reported APWV measurements 


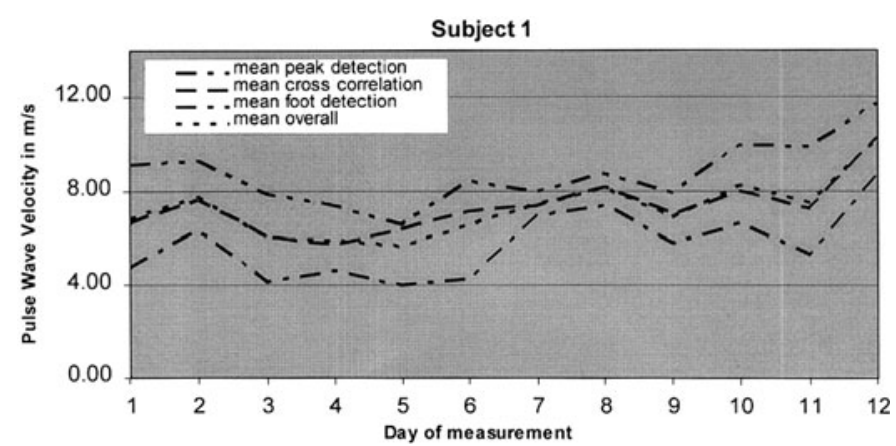

Figure 6. Comparison of three calculation methods for subject 1 .

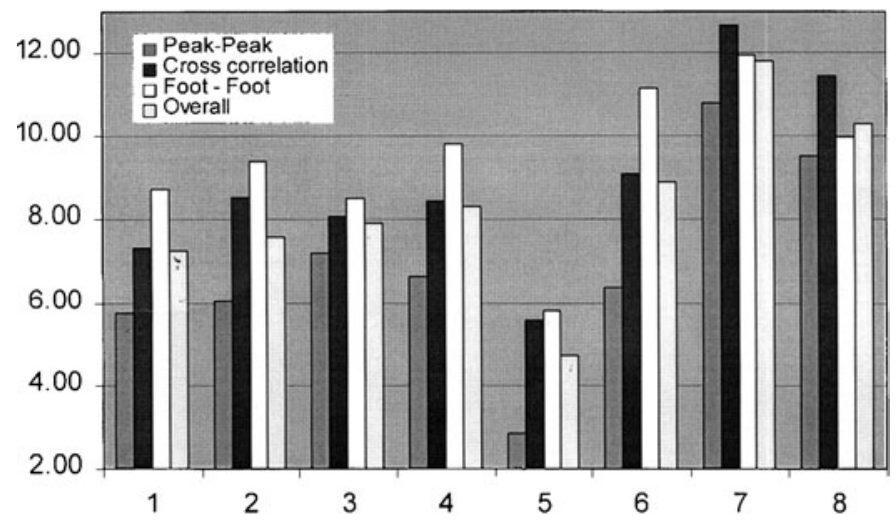

Figure 7. Histograms of PWV values for all three calculation methods for all eight subjects.

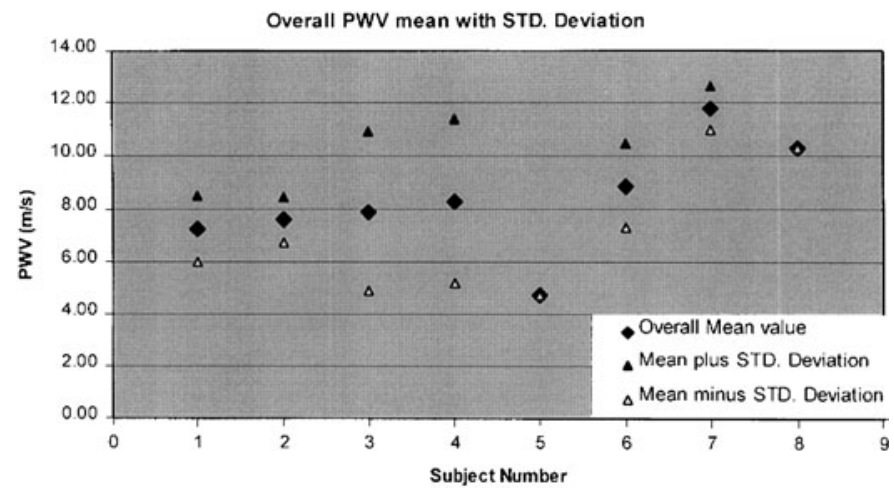

Figure 8. Overall mean values with \pm standard deviations for all eight subjects.

(Ramsey 1995, Wilkinson 1998, Lehman 2000). These results are all significantly higher than those reported (McCormack 1981) where ultrasound Doppler probes were used. The mean value reported in that work was $4.7 \pm 0.26 \mathrm{~m} \mathrm{~s}^{-1}$. 


\section{Discussion}

A comparison of the results using the three calculation methods (peak-to-peak; crosscorrelation; foot-to-foot) shows that the APWV values determined by the peak-to-peak detection method (PPAPWV) have the lowest mean standard deviation of $1.85 \mathrm{~m} \mathrm{~s}^{-1}$. However, the peak-to-peak detection method appears to give APWV values that are consistently lower than those obtained from the other two methods. The best results, then, are obtained by taking the mean of all three methods, leading to maximum accuracy and minimum variation.

The design and the selection of the digital filter to be used was a critical phase of this work. The program developed allows the user to implement four different IIR filter approximations: Butterworth, Chebyshev, Inverse Chebyshev and Elliptic. Extensive trials showed that a Butterworth lowpass filter with a cut-off frequency of $30 \mathrm{~Hz}$ gave the best results.

It must be emphasized that the APWV measurement technique developed in this work, applies to the peripheral arterial system and peripheral arterial disease. Only then can the PWV results be realistically applied to the Moens-Kortweg model to calculate the elastic modulus of the peripheral arterial segment under examination (brachial to radial in this work).

\section{Conclusions}

The object of the project was to develop a reliable and reproducible non-invasive method of measuring peripheral arterial pressure pulse wave velocity in humans, using piezoelectric pressure sensors. This included an analysis program which filters the measured data and calculates the arterial pulse wave velocity using three different methods: peak-to-peak detection, cross-correlation and foot-to-foot detection. The mean of all three results is taken as the most representative of the true pulse wave velocity. The values obtained are a little higher, but similar to values published in the literature.

The clinical usefulness of the instrument lies in the conversion of the velocity values to values of local stiffness (elasticity) of peripheral arterial walls. Patients with peripheral arterial disease, pre- and post-surgery patients (for example, bypass of superficial femoral block) and pre- and post-treatment patients (for example, urokinase for anterior tibial block) will be obvious beneficiaries of this non-invasive technique.

The next phase in the development of this instrument will involve such a clinical trial.

\section{References}

Lehman E D 2000 Aortic pulse wave velocity versus pulse pressure and pulse wave analysis Lancet $\mathbf{3 5 5} 412$ Malindzak G S and Meredith J H 1970 Comparative study of arterial transmission velocity J. Biomech. $3337-50$

McCormack P D 1981 Doppler Monitoring of the Peripheral Vascular Status of Human Subjects Under Variable Arterial Pressure (Pensacola, FL: Naval Aerospace Medical Research Laboratory)

Persson M, Eriksson H and Lindstrom K 2001 Estimation of arterial pulse wave velocity with a new tissue Doppler method Proc. 23rd Ann. Int. Conf. of IEEE Engineering in Medicine and Biology Society (Istanbul, 2001)

Moens S I 1878 Die Pulskurve (Leiden: Brill) pp 20-2

Ramsey M W 1995 Real time measurement of pulse wave velocity from arterial pressure waveforms Med. Biol. Eng. Comput. J. 33 636-42

Wilkinson I B 1998 Pulse analysis and arterial stiffness J. Cardiovasc. Pharmacol. 32 S33-7 